\title{
Einige Bemerkungen zu der Theorie von Weizsäcker über die Entwicklung der Riesensterne
}

\author{
Von Hans-Jost Binge* \\ (Z. Naturforschg. 6 a , 4953 [1951]; eing€gangen am 10. Januar 1951)
}

\begin{abstract}
C. F.v. Weizsäcker hat die Vermutung geäußert, daß die Kontraktion, welche nach dem Verlassen der Hauptreihe eintritt, bei Sternen hinreichender Masse so viel Energie freimacht, daß durch Strahlungsdruck eine sehr ausgedehnte Photosphäre entsteht und der Stern für lange Zeit als Roter Riese existiert.

In der vorliegenden Arbeit wird zunächst die Energie, welche beim Übergang eines Sterns in den Zustand eines Neutronentropfens frei wird, grob abgeschätzt und daraus die Lebensdauer der Roten Riesen größenordnungsmäßig abgeleitet.

Sodann wird die Stellung der Roten Riesen in offenen Sternhaufen und gewissen Arten von Doppelsternsystemen untersucht und gezeigt, daß sich diese am besten erklären läßt, wenn man annimmt, daß die Roten Riesen alt sind.

Zum Schluß werden die Typen von Veränderlichkeit diskutiert, welche bei Roten Riesen und intermediären Zwergen auftreten. Dabei wird eine volle Analogie zwischen beiden festgestellt.
\end{abstract}

$\mathrm{D}$ urch die Untersuchungen von v. We i z s ä c k e r über die Entwicklung der Sternsysteme und die daraus abgeleiteten Alterskriterien für Sterne ist es nahegelegt worden, daß es sich bei den Roten Riesen um ziemlich alte und nicht, wie früher angenommen, um die jüngsten Sterne handelt ${ }^{1}$. Daraus ergab sich, daß ihr innerer Aufbau von dem eines Hauptreihensterns wesentlich verschieden sein muß. Ferner zeigte J ordan ${ }^{2}$, daß bei einem Stern von einer Masse $M \geqq 1,44 M_{\odot}$ - diese Ungleichung ist für Rote Riesen erfüllt - nach Verlassen der Hauptreihe eine Kontraktion bis zur Dichte der Atomkerne einsetzen muß.

Nun ist die potentielle Gravitationsenergie einer homogenen Kugel von der Masse $M$ und der Dichte $\varrho$

$$
E_{\text {pot }}=-\frac{3}{5} \underset{R}{f M^{2}}=-\frac{3}{5} f M^{5 / 3} \sqrt[3]{\frac{4 \pi \varrho}{3}} .
$$

Bei einer Kerndichte von $6,6 \cdot 10^{13}$ ergäbe sich für $M=M_{\odot}=2 \cdot 10^{33} \mathrm{~g}$.

$$
\frac{\left|E_{\text {pot }}\right|}{M}=\frac{3}{5} f M^{2 / 3} \sqrt[3]{\frac{4 \pi \varrho}{3}}=4,1478 \cdot 10^{19} \mathrm{erg} / \mathrm{g} \approx c^{2} / 20 .
$$

Da nun die Masse eines Roten Riesen meistens größer als $2,8 M_{\odot}$ ist, so ergibt sich $\frac{\left|E_{\text {pot }}\right|}{M} \gtrsim c^{2} / 10$,

1 C. F. v. We iz säck er, Z. Astrophysik 24, 204 [1947]. d. h. die Kontraktion ist mindestens 14-mal ergiebiger als der Bethe-Prozeß. Ein Stern dieser Art hat nun eine Leuchtkraft von etwa 70 Sonnen und könnte also mehr als 40 Milliarden Jahre strahlen. Bei mehr als 20 Sonnenmassen und einer Leuchtkraft von 10000 Sonnen ergibt sich eine Lebensdauer von etwa 7 Milliarden Jahren.

Die äußeren Teile des kontrahierenden Sterns, dessen Kern infolge der Verkleinerung seiner Oberfläche immer heißer wird, werden durch Strahlungsdruck zu einer gewaltigen Hülle aufgebläht. Wenn diese Hülle für alles Licht, welches kurzwelliger als das infrarote ist, optisch dick ist, so wird der Stern uns das Bild eines Roten Riesen darbieten.

Es ist nun die Frage, wie die anderen Eigenschaften der Roten Riesen - v. Weizsäcker benutzt als Alterskriterien die schwache Rotation und das Vorkommen in der Population II - zu diesem Modell passen.

Da frühe Hauptreihensterne ihren Wasserstoff in rd. $10^{8}$ Jahren verbraucht haben, aber andrerseits die Spiralnebel für ihre Entwicklung einen Zeitraum gebraucht haben dürften, der nur wenig kleiner als das Weltalter $\left(5 \cdot 10^{9} \mathrm{Jahre}\right)$ ist, so muß innerhalb der offenen Sternhaufen, welche zur Population I gehören, ein Aussterben der O- und B-Riesen (vom oberen Ende der Hauptreihe) und ein Anwachsen der Zahl

* Hamburg 24, Graumannsweg 59.

2 P. J o rda n, Die Herkunft der Sterne, S. $41 \mathrm{ff}$. 


\begin{tabular}{|c|c|}
\hline Rote Riesen in Haufen & $\begin{array}{c}\text { Spektrum der hellsten } \\
\text { Haufensterne }\end{array}$ \\
\hline $\begin{array}{c}\text { keine } \\
\text { vereinzelte } \\
\text { wenige } \\
\begin{array}{c}\text { verhältnismäßig viele } \\
\text { sehr viele }\end{array}\end{array}$ & $\begin{array}{c}\text { O, B bis (A) } \\
\text { (O), B bis (A) } \\
(\mathrm{B}), \mathrm{A} \text { bis (F) } \\
\mathrm{A}\end{array}$ \\
\hline
\end{tabular}

Tab. 1.

der Roten und Gelben Riesen zu beobachten sein. Tatsächlich beobachtet man beim Übergang von Haufen ohne Rote Riesen zu solchen mit zahlreichen Roten Riesen ein Verschwinden der hellen O- und B-Sterne ${ }^{3}$, wie Tab. 1 zeigt.

An Hand dieser Beobachtungen ist es also wahrscheinlich, daß ein B-Stern der Hauptreihe, nachdem er in verhältnismäßig kurzer Zeit seinen Wasserstoff verbraucht hat, in den Zustand eines Roten Riesen übergeht.

Gegen diese Ansicht könnte man nun einwenden, daß es ebenso gut umgekehrt sein könnte und das Stadium eines Roten Riesen vor dem Stadium eines B-Sterns käme statt nach diesem. Dieser Einwand wird aber durch die Existenz der sog. ungleichartigen Doppelsterne, auf welche besonders $\mathrm{H} \mathrm{i} \mathrm{m} \mathrm{p} \mathrm{e}{ }^{4}$ hingewiesen hat, widerlegt. Es gibt nämlich neben Doppelsternen aus zwei Hauptreihensternen oder aus zwei Roten Riesen oder aus einem Hauptreihenstern und einem Roten Riesen auch solche aus einem Roten Riesen und einem Weißen Zwerg. Die bekanntesten Doppelsterne dieser Art sind $\alpha$ Scorpii und die beiden Mirasterne o Ceti und R Aquarii.

Ferner gibt es unter den wiederkehrenden Novae Doppelsterne, in denen der explosive O- bzw. BStern mit einem Roten Riesen bzw. Überriesen zusammen steht. Die bekanntesten Doppelsterne dieser Art sind T Cor. bor. und Z And. Nun läßt sich über die Novae und ihre Verwandten (U Gem.- und Z Cam.-Sterne) mit einiger Sicherheit sagen, daß diese Sterne in ihrem Ruhezustand zwischen der Hauptreihe und den Weißen Zwergen stehen ${ }^{5}$. Dabei ist nach $\mathrm{Miczaika}{ }^{6}$ die Entwicklung vermutlich folgende:

3 Ausführlicher bei W. Becker, Sterne und Sternsysteme, Th. Steinkopff, Dresden 1950, S. $154 \mathrm{f}$.

4 K. H im p el, Z. Naturforschg. 1, 417 [1946]; Urania, 11, 313 [1948]; Probleme der Entwicklung im Universum, C. Schwab, Stuttgart 1948, S. 90.

5 Vgl. u. a. K. H i m p e 1, Probleme der Entwicklung im Universum, S. 66, Abb. 7; Sternenwelt 2, 63 [1950] (Referat über Gasnebel und Neue Sterne, russisch); H. U. S a ndig, Sternenwelt 2, 158 [1950].

$$
\begin{aligned}
\text { Hauptreihe } & \rightarrow \text { Novae } \rightarrow \text { U Gem. } \rightarrow \text { ZCam. } \rightarrow \\
& \rightarrow \text { stabiler Weißer Zwerg. }
\end{aligned}
$$

Es ist aber anzunehmen, daß bei diesen Doppelsternen, genau so wie bei allen andern, beide Komponenten den gleichen Ursprung gehabt haben und folglich gleich alt sind. Nehmen wir einmal an, daß die Roten Riesen die jüngsten Sterne wären, so müßte auch der begleitende .Weiße Zwerg entsprechend jung sein. Da nun aber die blauen Sterne der Hauptreihe wegen ihres gewaltigen Wasserstoffverbrauchs jung sein müssen, so wäre - unter der Voraussetzung gleichen Ausgangsmaterials für alle Sterne - anzunehmen, daß bei Sternen wie $\alpha$ Scorpii B und o Ceti B oder den explosiven Komponenten von $\mathrm{T}$ Cor. bor. und $\mathrm{Z}$ And. eine chemische Zusammensetzung vorläge, welche etwa der von $\mathrm{Uns} \mathrm{ldd}^{7}$ ermittelten Zusammensetzung des Hauptreihensterns $\tau$ Scorpii entsprechen würde, daß sie also viel Wasserstoff enthielten. $\mathrm{Himpel}{ }^{8}$ nahm daher an, daß diese Art Sterne durch Kohlenstoff- und Stickstoffmangel zu Weißen Zwergen geworden seien und daß NovaAusbrüche bei ihnen mit dem Aufbau der Elemente und dem Einsetzen des B e the-Weizs äckerProzesses im Sinne der Eddingtonschen Nova Hypothese ${ }^{9}$ zusammenhingen.

Diese immerhin beachtliche Möglichkeit scheint aber nicht realisiert zu sein, da nach $M$ arsh a ${ }^{10}$ alle Weißen Zwerge an Wasserstoffmangel leiden und somit alt sind. Es bleibt also nichts anderes übrig, als die ungleichartigen Doppelsterne ebenfalls als alte Systeme zu betrachten. Damit wird aber auch die Frage nach dem Alter der Roten Riesen dahin beantwortet, daß diese älter sind als die frühen B- und O-Sterne der Hauptreihe. Es ist also auch die Frage nach der Herkunft der Roten Riesen in den offenen Sternhaufen eindeutig beantwortet.

Das Verschwinden der hellen und heißen O- und B-Sterne in Haufen mit vielen Roten Riesen bedeutet nichts anderes als die Umwandlung dieser Sterne in Rote Riesen, und demnach sind die Haufen mit vielen Roten Riesen wirklich älter als die Haufen mit frühen Hauptreihensternen.

Da die M-Komponenten der beiden ungleichartigen

6 G. M i c z a i k a, Z. Naturforschg. 2a, 219 [1947].

7 A. Unsöld, Z. Astrophysik 23, 75 [1943]; Z. Astrophysik 24, 278 [1947].

8 K. H i m p e l, Scientia [Milano] 38, 51 [1944]; Probleme der Entwicklung im Universum, S. 90.

9 A. S. E d d i n g t o n, Monthly Notices Roy. astronom. Soc. 99, 595 [1939].

10 R. E. M a r s h a k, Astrophysic. J. 92, 321 [1940]. 
Doppelsterne R Aquarii und o Ceti Mira-Sterne sind, so müssen die Mira-Sterne ebenfalls nach dem v. We i z s ä c k e r - J or d a n schen Modell aufgebaut sein. Das bedeutet aber doch, daß ihre eigentlichen Sternkörper den Weißen Zwergen ähnliche überdichte Sterne sind, welche von einer ausgedehnten Hülle umgeben sind.

Andrerseits zeigen aber gewisse Sterne unterhalb der Hauptreihe ebenfalls charakteristische Typen von Veränderlichkeit. Wie oben ausgeführt, sind die Novae sowie die U Gem.- und Z Cam.-Sterne solche Sterne.

Wenn also beide Gruppen von Veränderlichen im Grunde genommen den gleichen innersten Aufbau besitzen, so muß sich dies auch in gewissen verwandtschaftlichen Zügen offenbaren.

Das Charakteristikum der U Gem.- und Z Cam.Sterne ebenso wie das der Novae besteht darin, daß der Lichtwechsel dieser Sterne aus diskreten Ausbrüchen besteht, die durch explosive Vorgänge im Sterninnern hervorgerufen sind. Demgegenüber scheint es sich bei dem Mira-Lichtwechsel um eine gleichmäßige Pulsation des Sternes zu handeln. Aber nehmen wir nun einmal an, daß ein U Gem.-Stern, dessen „Periode“ etwa 340 Tage beträgt (UV Persei) in eine optisch dicke Hülle vom Durchmesser der Erdbahn eingebettet sei, dann würde die Energie des Ausbruchs zunächst von der Hülle absorbiert werden. Die Folge wäre dann ein Steigen der Temperatur und ein gleichzeitiges Expandieren der Hülle. Dieser Vorgang würde sich aber erst durch die ganze Hülle gewissermaßen hindurchfressen müssen und daher von außen viel langsamer in Erscheinung treten als bei einem „echten“ Weißen Zwerg ohne Hülle.

Da nun nach v. Weizsäcker ein Roter Riese aus einem massereicheren Stern entstanden ist als ein normaler Weißer Zwerg, so ist es evident, daß bei einer U Gem.-ähnlichen Veränderlichkeit des Riesen die bei einem Ausbruch freiwerdende Energie beträchtlich größer ist als bei einem U Gem.-Stern und daher ohne Schwierigkeit den Mira-Lichtwechsel unterhalten kann.

Es muß darauf hingewiesen werden, daß bei dieser Annahme die U Gem.- und die Mira-Sterne Parallelerscheinungen sind und daß kein U Gem.-Stern ein Mira-Stern werden kann.

11 Näheres bei H. Ludendorff, Handbuch der Astrophysik, Band VI, S. 113 ff., S. 159, S. 244. Springer, Berlin 1928.

12 G. M i c z a i k a, Himmelswelt 55, 65 [1948].

13 G. M i c z a i k a, Z. Naturforschg. 2a, 600 [1947].
Es ist nun interessant, daß es tatsächlich MiraSterne gibt, deren Lichtkurve erstaunliche Ähnlichkeit mit denen der U Gem.-Sterne besitzen. Diese Sterne sind die Mira-Sterne mit den längsten Perioden und dem rotesten Licht ${ }^{11}$. Je kürzer nun die Periode wird, desto mehr rücken die Ausbrüche aneinander, so daß zuletzt ein kontinuierlicher, sinusartiger Verlauf der Lichtkurve übrig bleibt. In diesem Fall tritt der nächste Ausbruch schon in Erscheinung, wenn der vorhergehende noch nicht abgeklungen ist, so daß die Amplitude des Lichtwechsels immer geringer wird und schließlich der Stern im konstanten Lichte leuchtet.

Bei den Mira-Sternen der Sonnenumgebung besteht nun nicht nur zwischen Form der Lichtkurve und Periodenlänge eine Beziehung, welche mit diesem Bilde übereinstimmt, sondern es zeigt sich, daß auch der Bewegungszustand der Mira-Sterne mit kurzer Periode in bezug auf das Milchstraßensystem ein ganz anderer ist als der der mit extrem langen Perioden und novaartigen Lichtkurven. Die MiraSterne mit sinusförmigem Lichtwechsel und kurzer Periode besitzen nämlich hohe Geschwindigkeit gegenüber der Gesamtheit der sonnennahen Sterne, während die Bewegung der U Gem.-artigen Mira-Sterne mit langer Periode im wesentlichen der Bewegung der sonnennahen Sterne entspricht ${ }^{12}$. Da die schnellbewegten Sterne in ihrer Gesamtheit (alle Sternarten zusammengenommen) ein Russell-Diagramm ergeben, welches der Population II entspricht, muß angenommen werden, daß die kurzperiodischen Mira-Sterne zur Population II gehören ${ }^{13}$. Andrerseits gehören die langperiodischen Mira-Sterne mit U Gem.-artigen Lichtkurven wegen ihrer geringen Abweichungen vom mittleren Bewegungszustand der Sonnen-Umgebung zur Population I.

Daraus folgt nach v. Weizsäcker, daß die MiraSterne mit kurzer Periode und sinusförmiger Lichtkurve erheblich älter sind als die langperiodischen mit novaartiger Lichtkurve. Aus diesen Erwägungen kann man folgende Rückschlüsse ziehen: die jüngsten Mira-Sterne sind die rotesten mit langen Perioden und novaartigem Lichtwechsel. Verlängert man diese Entwicklung weiter rückwärts. so müßte man zu Körpern kommen, bei denen immer katastrophalere Lichtschwankungen in immer längeren Intervallen stattfinden, d. h. zu einer Art Nova. Dieses hypothetische novaartige Vorstadium wäre wahrscheinlich der Übergang von den O- und B-Sternen der Hauptreihe zu den Roten Riesen. In diesem Stadium würde dann die Bildung der ausgedehnten Hülle vor sich 


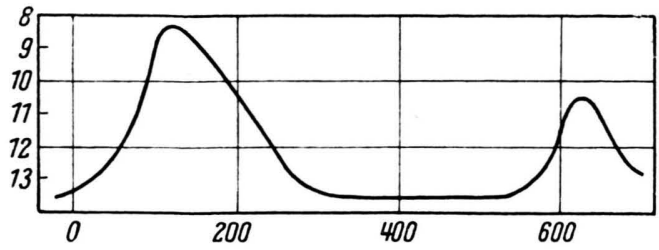

Abb. 1. Lichtkurve des Mira-Sterns Y Velorum - Periode 437 Tage - . Die Kurve erinnert mit ihrem langen konstanten Minimum an die einer wiederkehrenden Nova cder eines U Gem.-Sterns. (Aus Handbuch der Astrophysik, Bd. VI.)

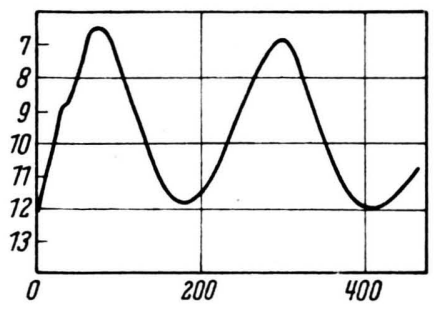

Abb. 2. Lichtkurve des Mira-Sterns R Bootis - Periode 223 Tage - . Typischer Fall eines kurzperiodischen MiraSterns mit sinusartiger Lichtkurve und guter Resonanz.

(Aus Handbuch der Astrophysik, Bd. VI.)

gehen, welche später den Kern des Roten Riesen wie eine Art Attrappe umgibt.

Die Energie, welche während dieser Katastrophenzeit ausgestrahlt wird, wird durch die Bildung des dichten Kerns geliefert. Dagegen wird die Strahlung des nachherigen Roten Riesen durch das allmähliche Niedersinken der Hülle auf den Kern erzeugt. Bei diesem sekundären Vorgang zieht sich der Stern zusammen und, weil dabei die Ausstrahlung konstant bleibt, aber die Oberfläche kleiner wird, wird der anfangs tiefrote Riesenstern außen wieder heißer und geht so in einen Gelben Riesen über.

Dabei nimmt die mittlere Dichte des Sterns zu und, da das Quadrat der Periode eines pulsierenden Sterns umgekehrt proportional seiner mittleren Dichte ist, so nimmt die Periode ab. Man kann also durch den oben skizzierten Entwicklungsvorgang den Zusammenhang zwischen Periodenlänge und Farbe einerseits und der Zugehörigkeit zur Population I oder II andrerseits auf Grund des v. Weizsäckerschen Modells der Roten Riesen erklären.

Wenn die Pulsation nicht mehr aus einzelnen U Gem.-artigen Stößen besteht, sondern gleichmäßiger wird, muß zwischen den Kern-Eruptionen und den Hüllenschwingungen Resonanz bestehen, wenn die Hülle „sauber“ mitschwingen soll. Da diese Be-

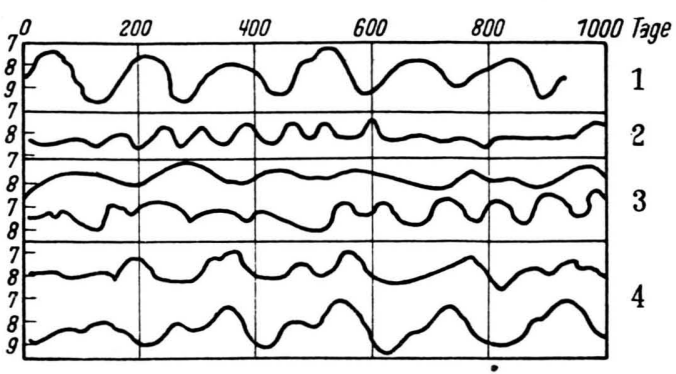

Abb. 3a. Lichtkurven von Roten Riesen mit halbregelmäßigem Lichtwechsel. X Monocerotis, V Ursae minoris, AF Cygni, $\mathrm{Z}$ Ursae majoris. (Aus L. C a m p b e ll u. L. J a c chia, „The Story of Variable Stars“, Harvard Books of Astronomy.)

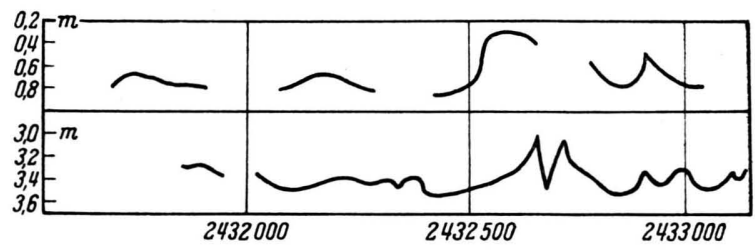

Abb. 3 b. Lichtkurven von Roten Riesen mit unregelmäßigem Lichtwechsel. $\alpha$ Orionis, $\alpha$ Herculis. (Aus W. Gliese, „Helligkeitsschätzungen veränderlicher Sterne ohne Hilfsmittel“. [Die Sterne, 26. Jahrg., Heft 1-3, S. 24].)

Zu Abb. 3 a und 3 b. Die Ähnlichkeit mit dem Lichtwechsel der Z Cam.-Sterne ist unverkennbar.

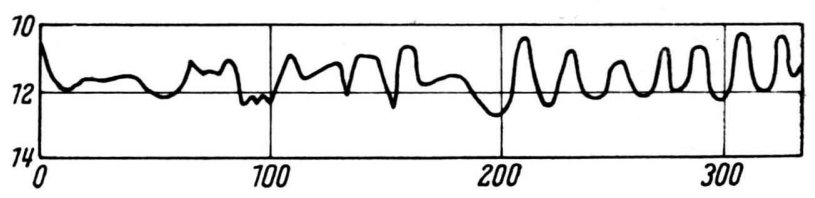

Abb. 4. Lichtkurve von Z Camelopardalis. Der Vergleich mit Abb. $3 \mathrm{a}$ und $3 \mathrm{~b}$ zeigt die große Ähnlichkeit eines gestörten U Gem.-Lichtwechsels mit der Veränderlichkeit der halbregelmäßigen und unregelmäßigen Roten Riesen. (Aus Handbuch der Astrophysik, Bd. VII.)

dingung nur selten über die ganze Lebensdauer des Riesen erhalten bleibt, so tritt häufig der Fall „unsauberer" Resonanz ein, die sich in einer unregelmäßigen Veränderlichkeit zeigt ( $\mathrm{J}$ o r d a n ${ }^{14}$ ). Dieses Phänomen tritt bei den $\gamma$-Kurven gewisser MiraSterne, bei den RV Tauri- und den $\mu$ Ceph.-Sternen auf. Wenn gar keine Resonanz mehr besteht, wird der Stern äußerlich konstant.

In Analogie zu dem obigen Schema von Miczaika ist also im Falle der Roten Riesen zu setzen:

14 P. Jordan, Die Herkunft der Sterne, S. 55. Wiss. Verlagsges. Stuttgart 1947. 
Helle Hauptreihen- $\rightarrow$ gewisse Novae? $\rightarrow$ Langperiodische sterne $(\mathrm{O}+\mathrm{B})$ Mira-Sterne ( $\alpha$-Kurven)

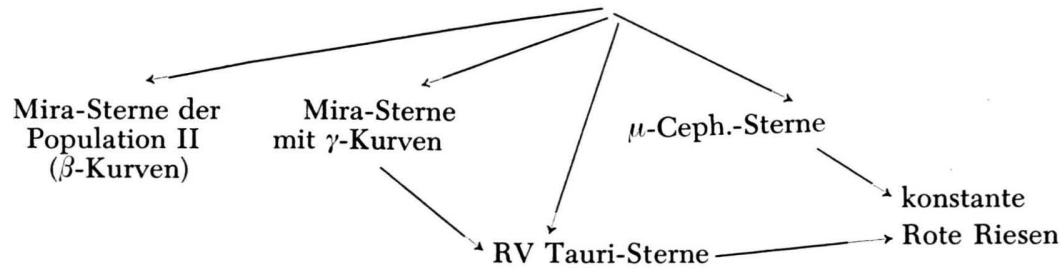

Daß die $\mu$ Ceph.- und RV Tauri-Sterne eine Stellung in der Entwicklung der Roten Riesen haben, der bei der Entwicklung der Weißen Zwerge das Z Cam.Stadium entspricht, ist durch die auffallende Ähnlichkeit der Lichtkurven dieser Riesen mit denen der Z Cam.-Sterne wahrscheinlich gemacht.

Im vorstehenden wurde gezeigt, daß man mit Hilfe der v. Weizsäckerschen Annahme über die Konstitution und Entwicklung der Roten Riesen interessante Einblicke in die Stellung dieser Sterne innerhalb von Sternsystemen, wie Doppelsternen, Stern- haufen und dem Milchstraßensystem, gewinnen kann. Außerdem wurde gezeigt, daß sich das Auftreten und die Entwicklung der Mira-Sterne zwanglos in das v. Weizsäckersche Bild der ,als Rote Riesen getarnten Weißen Zwerge“ einfügen lassen. Dieses Bild, welches v. Weizsäcker einzig und allein auf Grund seiner morphologischen Entwicklungskriterien (Kugelgestalt und schwache Rotation) und des Vorkommens in der Population II abgeleitet hat, hat sich also als Arbeitshypothese zur Erklärung der bei den Roten Riesen beobachteten Erscheinungen gut bewährt.

\section{NOTIZEN}

\section{Zum Kopplungsschema der Mesonen}

Von Walter Thirring

Max-Planck-Institut für Physik, Göttingen

(Z. Naturforschg. 6 a , 53-54 [1951]; eingeg. am 18. Januar 1951)

Bei dem Versuch, das $\mu$-Meson in die Theorie einzubauen, sind folgende zwei Kopplungsschemata diskutiert worden *:
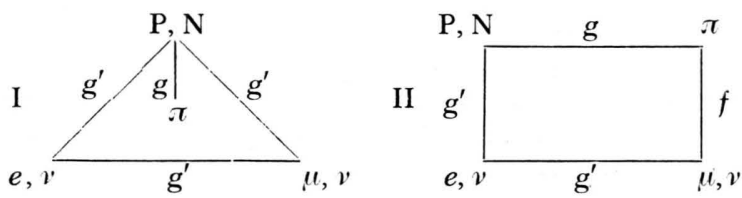

In Schema I lassen sich die drei Prozesse $\beta$-Zerfall, $\mu$ Zerfall und $\mu$-Einfang durch eine Kopplungskonstante $g^{\prime}$ beschreiben. Dies scheint eine gleiche Wechselwirkung aller Fermi-Téilchen untereinander anzudeuten analog der gleichen Kopplungskonstanten aller Teilchen mit dem elektrischen Feld, so daß g' dieselbe fundamentale Bedeutung wie $e$ beizumessen wäre. In Schema II gelingt es, die zwei Kopplungskonstanten $g$ und $f$ den drei Phänomenen, $\mu$-Einfang, Kernkräfte und $\pi$ - $\mu$-Zerfall, widerspruchsfrei anzupassen. Über letzteren Prozeß konnten bisher wegen Divergenzschwierigkeiten aus Schema I keine Auskünfte erhalten werden. Es soll hier kurz gezeigt werden, daß das aus Schema I folgende Verhältnis der

* Siehe H. Y uk aw a, Rev. mod. Physics 21, 474 [1949], wo sich weitere Literaturangaben finden.
Wahrscheinlichkeiten von $\pi$ - $\mu$ - und $\pi$-Elektron-Zerfall unabhängig von der Behandlungsweise divergenter Ausdrücke ist und leider zu Ungunsten des befriedigenderen Schemas I spricht.

Nach Schema I würde das $\pi$-Meson über ein virtuelles Nukleonenpaar in $\mu$-Meson und Neutrino oder Elektron und Neutrino zerfallen. Der Graph für den Prozeß sieht folgendermaßen aus:

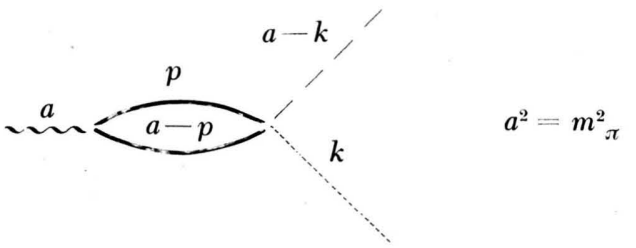

(Wellenlinie $\pi$-Meson, volle Linien Nukleonen, gestrichelte Linie $\mu$-Meson, punktierte Linie Neutrino. Die entsprechenden Energie-Impuls-Vektoren wurden über den Linien eingetragen.) Nach den bekannten Feynmannschen Regeln für die Berechnung der Elemente der S-Matrix läßt sich der Ausdruck für die Übergangswahrscheinlichkeit sofort als Integral im Impulsraum hinschreiben:

$$
\begin{gathered}
\left(\underline{p}=\underline{p}_{\mu} \gamma^{\prime \prime} \ldots\right) \\
\frac{1}{\tau_{\mu}}=\frac{g^{2} g^{\prime 2}}{(2 \pi)^{2} 2 m_{\pi}}\left[\int \frac{\mathrm{d} p}{(2 \pi)^{4}} \operatorname{Sp}[\underline{p}-M]^{-1}[\underline{p}-\underline{a}-M]^{-1}\right]^{2} \\
\cdot \int \mathrm{d} k \delta\left(k^{2}\right) \delta\left((a-k)^{2}-m^{2}{ }_{\mu}\right) \operatorname{Sp}\left(\underline{a}-\underline{k}+m_{\mu}\right) \underline{k} .
\end{gathered}
$$

